\title{
Changing FDA Approval Standards: Ethical Implications for Patient Consent
}

\author{
Jonathan J. Darrow, SJD, JD, MBA ${ }^{1,2}{ }^{\infty}$, Sanket S. Dhruva, MD, MHS ${ }^{3}$, \\ and Rita F. Redberg, MD, MSC ${ }^{3}$
}

'Program On Regulation, Therapeutics, And Law (PORTAL), Division of Pharmacoepidemiology and Pharmacoeconomics, Department of Medicine, Brigham and Women's Hospital and Harvard Medical School, Boston, MA, USA; ${ }^{2}$ Department of Law and Taxation, Bentley University, Waltham, MA, USA; ${ }^{3}$ Division of Cardiology, Department of Medicine, University of California, San Francisco School of Medicine, San Francisco, CA, USA.

J Gen Intern Med 36(10):3212-4

DOI: $10.1007 / \mathrm{s} 11606-021-06762-0$

(c) Society of General Internal Medicine 2021

$\mathrm{T}$ he pace of new drug and medical device introductions has accelerated in recent years. In 2018, 59 novel drugs were approved in the USA, the most since 1996. A rising proportion of drugs and devices qualify for one of the US Food and Drug Administration's (FDA) expedited programs, which allow approval based on less rigorous clinical trials. Expanded access and emergency use authorization allow access to products - such as remdesivir (Veklury) and COVID-19 vaccines - even before they are approved.

The growing array of products made available with limited evidence poses important challenges for patients and physicians. Ethical principles require that patients consent to treatment after being informed of the benefits and harms of each alternative. In routine practice, however, the consent process is often truncated, with limited presentation of alternatives, risks, and outcome data. As regulatory processes have evolved, the consent process - already criticized by some as inadequate - has changed little. We review the evolution of drug and device evidence requirements and consider the implications for informed consent.

\section{FDA APPROVAL PATHWAYS AND EVIDENCE REQUIREMENTS}

Following tragedies associated with thalidomide, the Dalkon Shield and other products, Congress strengthened premarket evidence requirements for drugs in 1962 and introduced them for medical devices in 1976. These laws helped ensure that products were safer and not fraudulent by requiring clinical testing before marketing, but also delayed availability, creating pressure to reduce requirements.

Received January 25, 2021

Accepted March 25, 2021

Published online April 8, 2021

\section{Drugs}

The 1962 Drug Amendments required evidence from adequate and well-controlled trials, and the traditional three phases of clinical trials were formalized in 1963 as a way of systematically demonstrating efficacy and safety. The FDA later outlined characteristics of trials, such as randomization and blinding, that could help to meet the statutory standard.

But in the years that followed, pressure mounted for the agency and Congress to condense evidence requirements. The 1983 Orphan Drug Act encouraged the FDA to interpret evidence requirements flexibly when evaluating rare disease medications (Fig. 1). More specific flexibilities in endpoints and the number and phase of trials were also introduced. For example, in 1988, the fast-track program was created to permit approval of high-priority medicines on the basis of earlierstage trials, and a 1992 accelerated approval program expanded the use of surrogates of efficacy (such as laboratory values believed to correlate with patient-relevant endpoints) that were only "reasonably likely" to predict clinical benefit. A 1997 law authorized approval on the basis of single clinical trial. In 2012, the Breakthrough Therapy designation further relaxed requirements by allowing reliance on pharmacodynamic biomarkers that would "not meet criteria for an acceptable surrogate endpoints" under the accelerated approval standard, ${ }^{1}$ and encouraged approval based on "fewer, smaller, or shorter clinical trials." 2 Most recently, Congress and the FDA have promoted the use of "novel clinical trial designs," real-world evidence (to help support the approval of secondary indications), "non-traditional development programs," and "new approaches to statistical analysis." 3

These programs have supported approval of a few highly effective drugs, such as imatinib (2001) for chronic myelogenous leukemia, which used the Orphan Drug Act, fast-track, and accelerated approval programs, and sofosbuvir (2013) for chronic hepatitis $\mathrm{C}$ virus infection, which used the fast-track and breakthrough therapy programs. In most cases, however, drugs approved under these programs offered clinical benefits that were either small, based on limited data, or comparable to existing products. ${ }^{4}$ For example, eteplirsen (Exondys 51), a drug for Duchenne muscular dystrophy, was approved based 


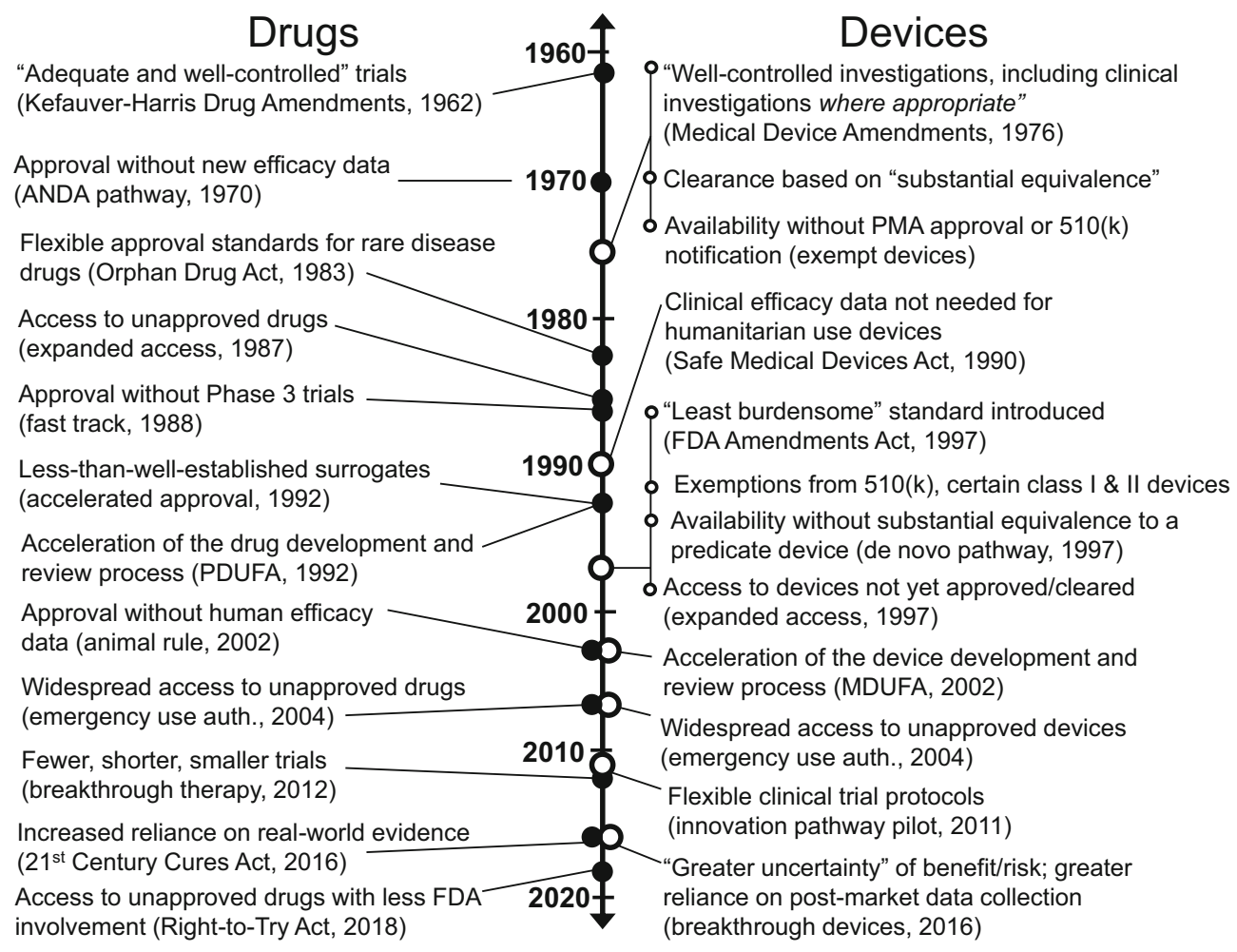

Figure 1 The evolution of evidence requirements for new drugs and devices, 1960-2020. ANDA, abbreviated new drug application; PMA, premarket approval application; PDUFA, Prescription Drug User Fee Act; MDUFA, Medical Device User Fee Act.

on increases in a biomarker (dystrophin levels) so small that meaningful patient benefit was improbable.

\section{Medical Devices}

Regulatory requirements that medical devices provide "reasonable assurance of safety and effectiveness" have become easier to satisfy. In 1990, Congress created the Humanitarian Device Exemption, allowing approval without reasonable assurance of effectiveness (based on safety and probable benefit) when targeted diseases affect fewer than 4,000 people in the USA (8,000 since 2016). A 1997 law expanded exemptions from premarket review to include most low- and some moderate-risk devices, created a streamlined "de novo" pathway for novel devices thought to not be high-risk, and directed FDA to require the "least burdensome" means of satisfying regulatory standards. The 2016 21st Century Cures Act reinforced this approach, encouraging post-approval evidence collection to reduce some pre-approval requirements, and creating a Breakthrough Devices designation that allowed the FDA to "accept a greater extent of uncertainty" for a broad range of devices. ${ }^{5}$

\section{IMPLICATIONS FOR PHYSICIAN-PATIENT-INFORMED CONSENT PROCESS}

Allowing marketing based on more limited evidence means that patients are increasingly exposed to treatments earlier in the evidence development process. But early promise based on limited evidence may not translate to meaningful benefit. For example, the FDA published a report of 22 cases in which early-stage positive results were not found in phase 3 trials. ${ }^{6}$ Patients and physicians also may be unaware that data generated during routine clinical care are increasingly used for regulatory decision-making, blurring the distinction between research and treatment.

These changes create a need to update the process of obtaining informed treatment consent. As treatment and research converge, physicians' communication of benefit-risk information to patients should borrow elements traditionally used to protect clinical trial subjects, such as plain language written disclosure of risks, benefits, and alternatives.

The FDA has provided a model for product-specific consent with the Essure sterilization implant, which was approved based on limited evidence and later found to be associated with complications such as tubal perforations and unintended pregnancies. In 2016, the FDA issued a guidance document that included an information checklist for patients and physicians to sign when discussing risks and benefits. Absent FDA action, physicians, health systems, and professional societies should take action to facilitate written, product-specific disclosure to patients similar to Vaccine Information Statements, including quantitative outcome measures and remaining uncertainties. Such signed documents would augment current disclosures for medical devices, and would be applied to drugs approved through expedited pathways.

Such new disclosure documents should be complemented by improved labeling that provides a clear summary ("facts box") of available data, similar to 
nutrition labeling. Existing product labels are often lengthy, with salient data about risks and benefits mixed with less important information. For example, concerns over cardiovascular events led to helpful, columnar outcome data in the labeling for rosiglitazone (Avandia), but data were scattered across the 42-page label. A summary "facts box" could be paired with a "red box" that employs icons that highlight at a glance which key trial characteristics are lacking, such as a crossed-out mask to indicate the absence of blinding or a laboratory flask to indicate reliance on surrogate endpoints. Recent FDA efforts to summarize approval data in online Drug Trials Snapshots could be improved by better translating esoteric outcome data to plain language descriptions of how trial subjects felt, functioned, or survived, and could be expanded to include medical devices.

\section{CONCLUSION}

The public trusts the FDA to approve only meaningfully beneficial products, but pressure to expedite approvals after the 1962 and 1976 laws has led to increasing abridgment of the testing process. Speedier availability is sensible when products offer substantial improvements but should not be driven by disease severity alone. When expedited approvals occur, physicians have the responsibility to clearly communicate known benefits, risks, and uncertainties, along with data about the various alternatives, to safeguard informed consent and patient autonomy and guide patients to the most promising options.

Acknowledgments: The authors thank Aaron Kesselheim for contributing to the development of this paper.

Corresponding Author: Jonathan J. Darrow, SJD, JD, MBA; Program On Regulation, Therapeutics, And Law (PORTAL), Division of Pharmacoepidemiology and Pharmacoeconomics, Department of Medicine, Brigham and Women's Hospital and Harvard Medical School, Boston, MA, USA (e-mail: jjdarrow@bwh.harvard.edu).

Funding The authors are supported by a Greenwall Foundation Making a Difference grant. Dr. Darrow is also supported by grants from Arnold Ventures and the Kaiser Permanente Institute for Health Policy, and by a Novo Nordisk Foundation grant for a scientifically independent Collaborative Research Programme (grant NNF17SA0027784). Drs. Redberg and Dhruva are supported by a grant from Arnold Ventures. Dr. Dhruva is supported by the National Evaluation System for Health Technology Coordinating Center (NESTcc) and the National Heart, Lung, and Blood Institute (NHLBI) of the National Institutes of Health (NIH) (K12HL138046). The funders had no role in the design and conduct of the research, the review or approval of the manuscript, or the decision to submit the manuscript for publication.

\section{Declarations:}

Conflict of Interest: The authors declare that they do not have a conflict of interest.

\section{REFERENCES}

1. FDA. Breakthrough therapy. Available at https://www.fda.gov/patients/ fast-trackbreakthrough-therapy-accelerated-approval-priority-review/ breakthrough-therapy. Accessed 6 January 2021.

2. FDA. Guidance for industry: expedited programs for serious conditions: drugs and biologics. May 2014.

3. PDUFA reauthorization performance goals and procedures fiscal years 2018 through 2022. 163 Cong. Rec. S4741. August 2, 2017.

4. Darrow JJ. Few new drugs deserve expedited regulatory treatment. J Managed Care Spec Pharm. 2021. (in press). Available at https://papers. ssrn.com/sol3/papers.cfm?abstract_id $=3745284$. Accessed 6 January 2021.

5. Johnston JL, Dhruva SS, Ross JS, Rathi VK. Early experience with the FDA's Breakthrough Devices program. Nature Biotech. 2020;38:933-8.

6. FDA. 22 case studies where phase 2 and phase 3 trials had divergent results. January 2017. Available at https://www.fda.gov/media/102332/ download. Accessed 6 January 2021.

Publisher's Note: Springer Nature remains neutral with regard to jurisdictional claims in published maps and institutional affiliations. 